\title{
Correlation Between Static and Dynamic Parameters of A-to-D Converters: In the View of a Unique Test Procedure*
}

\author{
F. AZAÏS, S. BERNARD, Y. BERTRAND, M. COMTE AND M. RENOVELL \\ LIRMM-CNRS/University of Montpellier, 161, Rue Ada, 34392 Montpellier, Cedex 5, France
}

Received September 9, 2002; Revised May 12, 2003

Editors: F. Vargas and V. Champac

\begin{abstract}
ADCs are fully characterized by both static and dynamic parameters. Testing methods usually combine a histogram-based approach with a spectral analysis to determine the complete set of ADCs parameters. In the view of a unique test procedure, this paper investigates the correlation between both kinds of parameters. Experimental results demonstrate that under appropriate test conditions, the dynamic parameters extracted from a classical FFT exhibit significant variations against ADC offset, gain and non-linearity errors, opening the way of a low-cost test strategy in the frequency domain.
\end{abstract}

Keywords: analog and mixed-signal testing, ADC test, spectral analysis

\section{Introduction}

At present, most part of the signal processing in areas like instrumentation, telecommunications, control and consumer electronics is carried out at the digital level. The role of Analog-to-Digital Converters (ADCs) placed at the borders of the digital domain is getting a particular relevance, since the signal degradation introduced by these components cannot normally be recovered by subsequent processing. The correct evaluation of the whole system performance therefore requires the test of these mixed-signal devices. As the new generations of ADCs provide increasing speed and resolution, test requirements become more and more stringent, resulting in ever more expensive test procedures. The test cost frequently reaches half the price of the device itself. The markets of both stand-alone ADCs and ADC macrocells to be embedded in complex ASICs would benefit from the availability of low-cost test methods.

*This work has been selected from the 3rd IEEE Latin-American Test Workshop (LATW2002).
The industrial testing of ADCs is still largely specification based. Parameters listed in the specification of a converter can actually be divided in two groups, one related to the transfer function and another that expresses the deformation induced on the converted signal. In the first group, one finds the so-called static parameters such as offset, gain, differential and integral non-linearity errors. These parameters are usually evaluated using a histogram-based approach $[6,7]$, which is based on a statistical analysis of how many times each code appears on the ADC output for a given input signal. The main disadvantage of this technique resides in the high number of samples required to achieve statistically satisfactory results. The required number of samples can become unacceptably large for high resolution converters, especially in presence of noise. Even present day Automated Test Equipment (ATE) has problem with the efficient handling and processing of such large sample sets. The second group of ADC parameters is composed of the so-called dynamic parameters. Examples of such characteristics are the Signalto-Noise And Distortion ratio (SINAD), the SpuriousFree Dynamic Range (SFDR) or the Total Harmonic 
Distortion (THD). These parameters are usually evaluated from a Fast Fourier Transform (FFT) of the digital samples acquired on the converter output when a pure sine-wave is applied to its input [9]. Relatively small sample sets are usually sufficient to get good estimates of the ADC dynamic parameters. Complete characterization of a converter can therefore be obtained by coupling a histogram-based approach with a spectral analysis [4]. Finding a link between static and dynamic parameters could enable one to deduce the complete set of an ADC parameters from a unique test acquisition and processing [2]. Spectral analysis would be preferable to the histogram method for two reasons [8]: firstly, it is more representative of the converter running reality, and secondly it requires less samples, which means a shorter processing time and reduced storage resources, two major considerations in the perspective of low-cost ADC testing. Hence, it is the objective of this work to investigate whether the dynamic parameters are sensitive to ADC static errors and under which test conditions.

The paper is organized as follows. Section 2 defines the dynamic parameters and Section 3 presents the experimental setup. Section 4 gives a preliminary study on the sensitivity of the considered dynamic parameters upon test conditions: the number of samples taken into account for the FFT, the number of input signal periods during acquisition and the input signal amplitude are successively treated. Then, the influence of static parameters on dynamic performances is explored in Section 5. Section 5.1 sets out the impact of an offset error, while the consequences of a gain error are presented in Section 5.2. The study of non-linearity errors is more complex than the one of other static errors. Thus, Section 5.3 is divided in two parts: firstly a study of influence of a single non-linearity on one code, secondly the influence of different shapes of global nonlinearity. Finally, Section 6 gives some concluding remarks.

\section{Definitions}

Spectral analysis of ADC is based on the exploitation of the Fourier transform of the numerical samples acquired on the converter output, when a pure sine-wave is applied to its input. While the spectrum of a pure sinewave consists of a unique line located at the frequency of the signal, the spectrum of the corresponding quantized output through an ideal converter comprises noise components throughout the frequency range. These parasitic elements are due to the quantization principle itself, that induces predictable quantization noise. In the case of a real converter affected by non-idealities, additional noise and harmonics of the signal frequency also appear in the spectrum. Therefore, the analysis of a real converter is conducted in comparison with the one of a perfect $\mathrm{ADC}$, in order to differentiate the intrinsic quantization noise from the one resulting of the physical device imperfections. Spectral test actually consists in processing the frequency components of the spectrum to determine the different test parameters listed below.

The Signal-to-Noise Ratio (SNR) of an ideal ADC is defined by the ratio of the input signal effective value over the quantization noise effective value. A good estimation of the $S N R$ value for a perfect $n$-bit converter excited by a full scale signal is given by [5]:

$$
S N R_{\mathrm{dB}}=6.02 \times n+1.76
$$

As mentioned, real converters generate extra noise and harmonics, which have to be considered in the ADC performances. Consequently, the corresponding parameter dedicated to real converters is the SINAD (SIgnal-to-Noise And Distortion ratio), extracted from the spectrum as follows [5]:

$$
\operatorname{SINAD}_{\mathrm{dB}}=20 \times \log _{10}\left(\frac{S}{\sqrt{\sum_{f \neq f_{\mathrm{in}}} S_{i}^{2}}}\right)
$$

where $S$ is the input signal effective value, and $S_{i}$ the effective value of the $i$ th harmonic component.

When the input signal peak-to-peak amplitude $2 A_{\text {in }}$ differs from the converter full scale $F S\left(2 A_{\text {in }} \leq F S\right)$, a corrective term is applied to the SINAD to enable comparisons and calculation of the effective number of bits [5]:

$$
S I N A D_{\mathrm{FS}}=\operatorname{SINAD}_{2 A_{\mathrm{in}}}+20 \times \log _{10}\left(\frac{F S}{2 A_{\text {in }}}\right)
$$

The effective number of bits, $n_{\text {eff }}$, is a widely used performance criteria, derived from the expression of the SNR [5]:

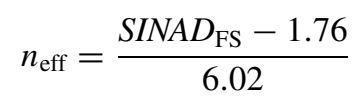

This sets out the ADC effective resolution, that means the equivalent number of bits a perfect $\mathrm{ADC}$ would 


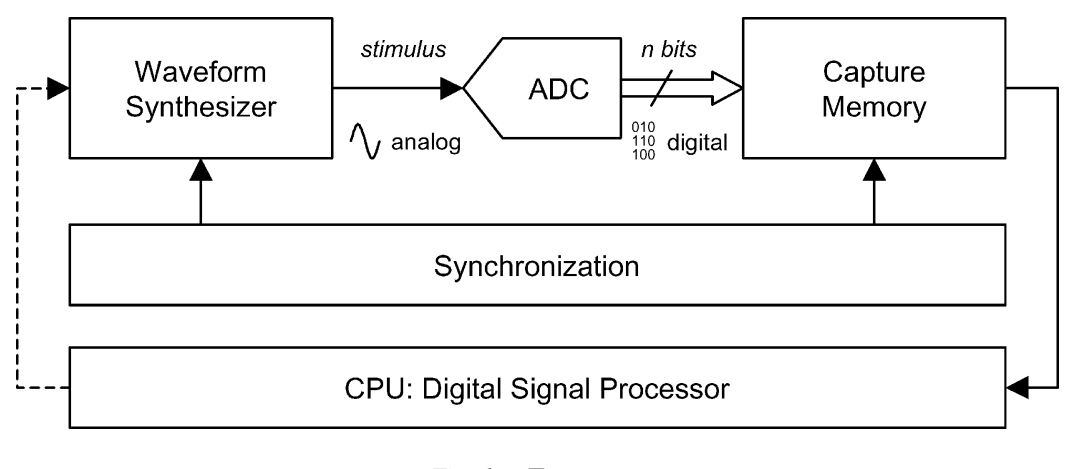

Fig. 1. Test setup.

have if all the noise observed on the spectrum was due to quantization process.

To give an indication upon the harmonic distortion, particularly needed in the audio field, the THD (Total Harmonic Distortion) is determined from the harmonic compoentss $H_{k}$ located at multiple frequencies of the input signal in the Nyquist band (from 0 to half the sampling frequency) [5]:

$$
T H D_{\mathrm{dB}}=20 \times \log _{10}\left(\frac{\sqrt{\sum_{k} H_{k}^{2}}}{S}\right)
$$

In practice, only the first few harmonics of the output signal are actually treated as distortion, while the remaining distortion is treated as noise. In this paper, we will differentiate the Total Harmonic Distortion computed with all harmonics in the Nyquist band from the $T H D_{1-5}$ computed only with the first five harmonics.

The SFDR, Spurious Free Dynamic Range, is the ratio of the greatest spectral component, which may appear anywhere in the spectrum and not necessarily in an harmonic bin, over the signal [5]:

$$
S F D R_{\mathrm{dB}}=20 \times \log _{10}\left(\frac{\max \left(S_{i}\right)}{S}\right)
$$

The floor level of noise represents the average level of noise excluding the fundamental and the harmonics considered in the $T H D_{1-5}$. Many definitions can be found in the literature for this parameter. In this paper, we adopt the one that is the closest to the noise mean power:

$$
B_{m}=20 \times \log _{10}\left(\frac{\sqrt{\sum_{f \neq f_{\text {in }}} S_{i}^{2}-\sum_{k=1}^{5} H_{k}^{2}}}{\frac{N}{2}-7}\right)
$$

\section{Experimental Setup}

The typical test setup for ADC dynamic testing on a classical ATE is illustratedFig. 1. The waveform synthesizer generates a sine-wave signal with an input frequency $f_{\text {in }}$, an amplitude $A_{\text {in }}$ and an offset $V_{o}$. This stimulus is applied on the converter input and the resulting samples on the converter output are acquired in the capture memory at the rate of the sampling frequency $f_{s}$. These samples are then transferred to the CPU for further processing: dynamic parameters are extracted from a FFT on the digital sample set. Note that coherent sampling is usually used to guarantee that each sample carries unique and independent information. Coherence consists of an integer number $N$ of samples acquired at frequency $f_{s}$ that are equally spaced over an integer number $M$ of identical signal periods at frequency $f_{\text {in }}$, with $N$ and $M$ relatively prime. When coherent sampling is used in the frequency analysis domain, the stimulus fundamental component and each of its harmonics fall precisely on single frequencies of the spectrum. This fact allows an easier location and a more precise power measurement, which then leads to better results for the parameter measurements. Implementing coherence on ATE requires synchronization between the synthesizer, the ADC under test and the capture memory.

This experimental setup has been implemented on the HP VEE software, a measurement programming environment that can be used for both simulation and physical test. For simulation, we consider a classical model of ADC presenting a stair shaped transfer function. In case of an ideal $n$-bit ADC, the transfer function manifests $\left(2^{n}-1\right)$ equally spaced transition levels over the full scale $(F S)$ range of the converter. The width of a step is a quantum or Least Significant Bit ( $L S B)$, 
given by:

$$
q=1 \quad L S B=\frac{F S}{2^{n}}
$$

In case of a real converter, the transfer function is affected by some non-idealities characterized by the static parameters. An offset error can be simply modeled by adding (or subtracting) the same quantity to all transition levels, resulting in an horizontal shift of the ideal transfer function. A gain error is modeled by multiplying all transition levels by the same factor, resulting in a compression or dilation of the ideal transfer function. Non-linearity errors are modeled by individual variations of the transition levels, resulting in a deviation of the actual transfer function from the ideal one.

\section{Dynamic Parameters vs. Test Conditions}

In order to determine whether static errors have an influence on the dynamic test parameters measured through spectral analysis, it is important to evaluate the sensitivity of these dynamic parameters to the test conditions. Investigations have been performed varying the number of samples $N$ considered to perform the FFT, the number of periods $M$ of the input sinewave during acquisition and the input signal amplitude $A_{\text {in }}$. Results presented in this section are based on a 6-bit ADC but remain valid for higher resolution ADCs.

\subsection{Influence of the Number of Samples}

The number of samples taken into account for the analysis is an important factor to consider for low-cost testing, since large records necessitate more acquisition and computation time and larger storage memory. For illustration, Fig. 2 givesthe measured SINAD and $B_{m}$ for several input signals presenting a small phase shift. Regarding the SINAD parameter, it clearly appears that the measured value does not depend on the number of samples provided it is high enough. Similar results have been observed for all dynamic parameters, except $B_{m}$. Hence, we will use 1024 samples for each FFT computation afterwards in order to leave out of account the uncertainty on the synchronization. Regarding the mean level noise $B_{m}$, the measured value decreases as the number of samples gets higher but appears not really sensitive to an input signal phase shift. Addi-
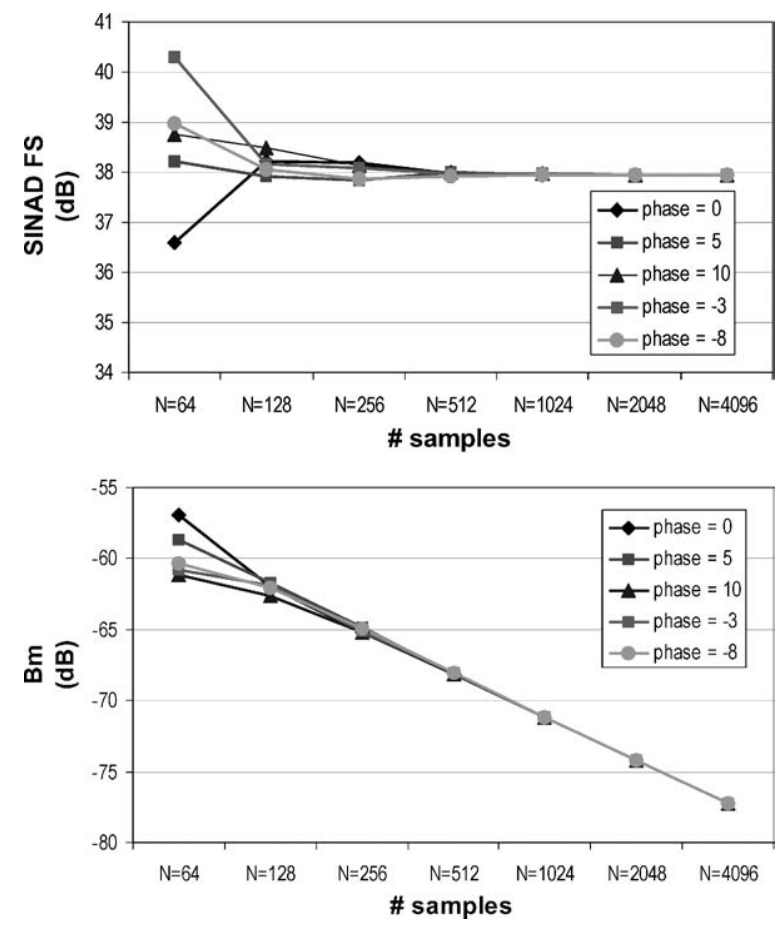

Fig. 2. Dynamic parameters vs. number of samples.

tional experiments have pointed out that this parameter does not depend on other factors than the number of samples used for the FFT computation. Therefore, in further sections, we will not present the study of its invariability versus the setup conditions.

\subsection{Influence of the Number of Input Periods}

Spectral analysis is generally performed at ADC maximal sampling frequency and nominal input signal frequency. The number of periods is adjusted to collect the required sample set with respect to the coherence sampling condition since it is widely accepted that the number of periods of the input signal has no impact on the measured parameter values. In practice, we found out that this number of periods only alters the THD computed using all the harmonics in the Nyquist band (seeFig. 3). Indeed, when the number of periods increases, extra bins appear between the harmonic bins. As the number of bins is a constant equal to $N / 2$, the number of harmonics considered in the Total Harmonic Distortion value decreases. In contrast, a stable value is measured whatever the number of periods if the $T D H$ is evaluated using only a limited number of harmonics. 


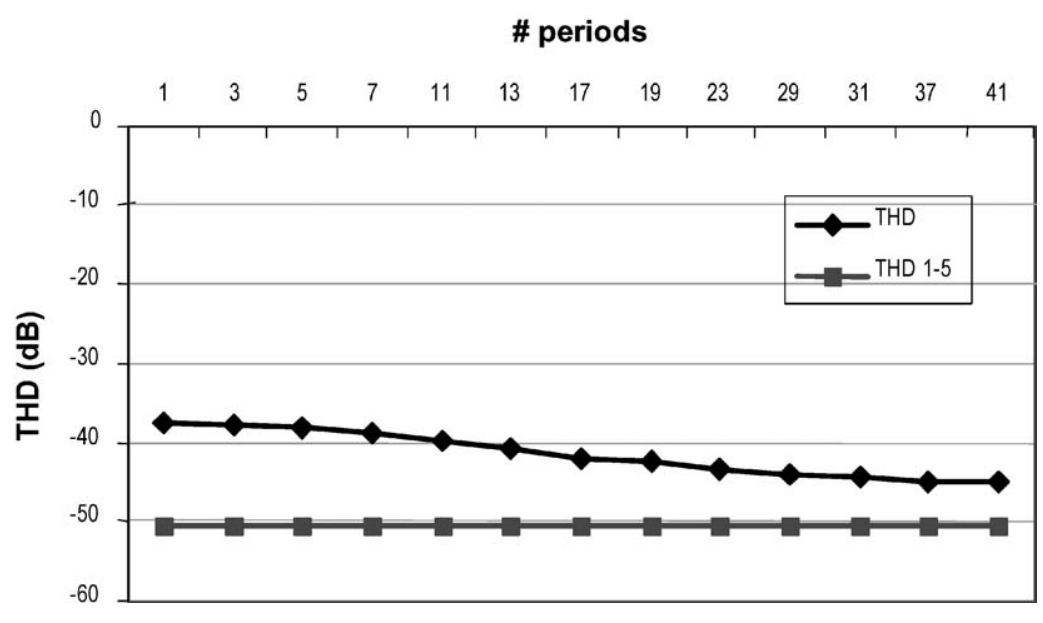

Fig. 3. Dynamic parameters vs. number of periods.

\subsection{Influence of the Input Signal Amplitude}

In a classical testing environment, it is extremely difficult to precisely guarantee the value of the generated input signal amplitude. Nevertheless, the values of all the dynamic parameters except $B_{m}$ depend on this signal amplitude. In order to evaluate the potential error on the extracted parameters, experiments have been conducted to determine the maximal variation of the dynamic parameters for a large range of input signal amplitudes.

Spectral analysis is usually performed using a sinewave with a peak-to-peak amplitude slightly lower than the full scale of the ADC. Fig. 4presents the evolution of the ADC parameters for decreasing amplitudes from full scale. The SINAD and SFDR are sensitive to amplitude variations but in a tolerable range. A total variation of $0.6 \mathrm{~dB}$ is observed for the $\operatorname{SINAD} D_{\mathrm{FS}}$, which corresponds to a relative accuracy of $1.6 \%$. In the same way, a total variation of $2.8 \mathrm{~dB}$ is observed for the $S F D R$, which corresponds to a relative accuracy of $4.6 \%$. In contrast, the $T D H_{1-5}$ presents an extreme sensitivity with a total variation higher than $30 \mathrm{~dB}$. A deviation in the input signal peak-to-peak amplitude less than 0.1 quantization step $(0.1 \mathrm{LSB})$ can actually result in a variation of $20 \mathrm{~dB}$ or more in the measured harmonic distortion, corresponding to a relative accuracy of $50 \%$. Amplitude deviations of this size are quite common in a production environment, where deviations around $1 \%$ may occur, yielding a deviation of more than one and a half quantization step in a 6-bit converter [3]. The present-day solution to this sensitivity problem is noise dithering [1]. But this technique has several drawbacks, in particular the relatively high number of samples required to achieve a significant reduction of the sensibility of the THD and the influence of the noise dither on the SINAD parameter.

In contrast to conventional dynamic test, one can apply a signal with a peak-to-peak amplitude slightly higher that the ADC full scale. Fig. 5 presentsthe evolution of the ADC parameters for increasing amplitudes from full scale. The total variation observed in this case appears quite important but much more predictable. For instance considering an input signal with a peakto-peak amplitude 2 LSB higher than $F S$, a deviation of $\pm 0.1 \mathrm{LSB}$ in the amplitude results in a variation of about $0.4 \mathrm{~dB}$ in the SINAD, $0.7 \mathrm{~dB}$ in the SFDR and $0.8 \mathrm{~dB}$ in the $T D H_{1-5}$, corresponding to relative accuracy of $1.3 \%, 2 \%$ and $2.2 \%$ respectively. The relative accuracy for a given amplitude deviation around a nominal point is therefore greatly improved by the use of an input signal with an amplitude higher than full scale, even if measured values do not directly represent the ADC performances.

\section{Dynamic Parameters vs. Static Errors}

In order to evaluate the influence of static errors on the dynamic parameters, experiments have been conducted introducing various errors in the converter model, in particular offset, gain and non-linearity errors. Note that the previous section has stated out that the SINAD, SFDR and $T H D_{1-5}$ parameters are not sensitive to the number of samples (provided that it is high enough) neither to the number of input periods. Hence, 


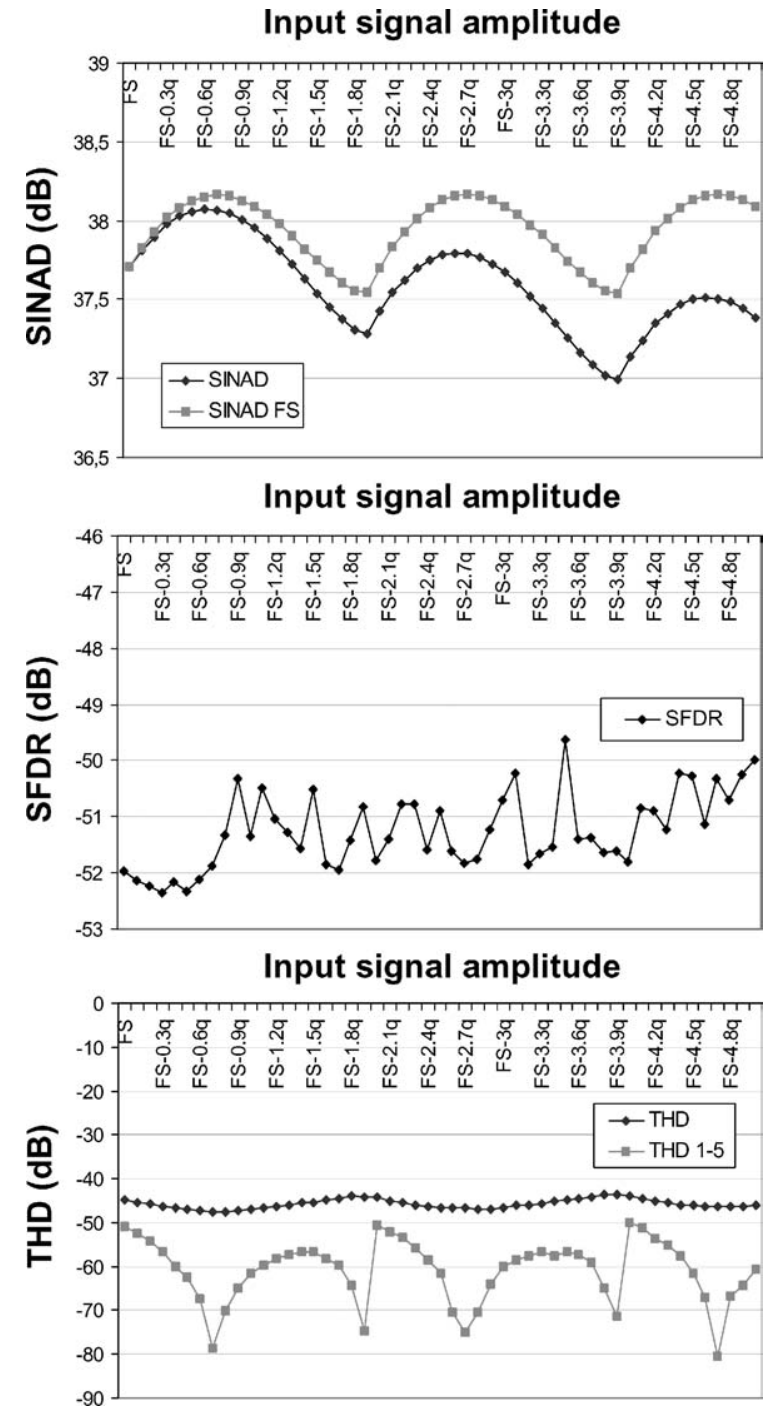

Fig. 4. Dynamic parameters vs. signal amplitude $\left(2 A_{\text {in }} \leq F S\right)$.

investigations on the influence of static errors on dynamic parameters can be performed independently of these test conditions. In this study, we use $N=1024$ and $M=31$.

At the opposite, the SINAD, SFDR and $T H D_{1-5}$ parameters are sensitive to any amplitude deviation of the input signal. This is particularly true for the $T H D_{1-5}$ when the input signal peak-to-peak amplitude is lower than the full scale range of the converter. This sensitivity has therefore to be taken into consideration when studying the impact of static errors on dynamic parameters. In particular, one should differentiate between the case of a signal peak-to-peak amplitude lower and
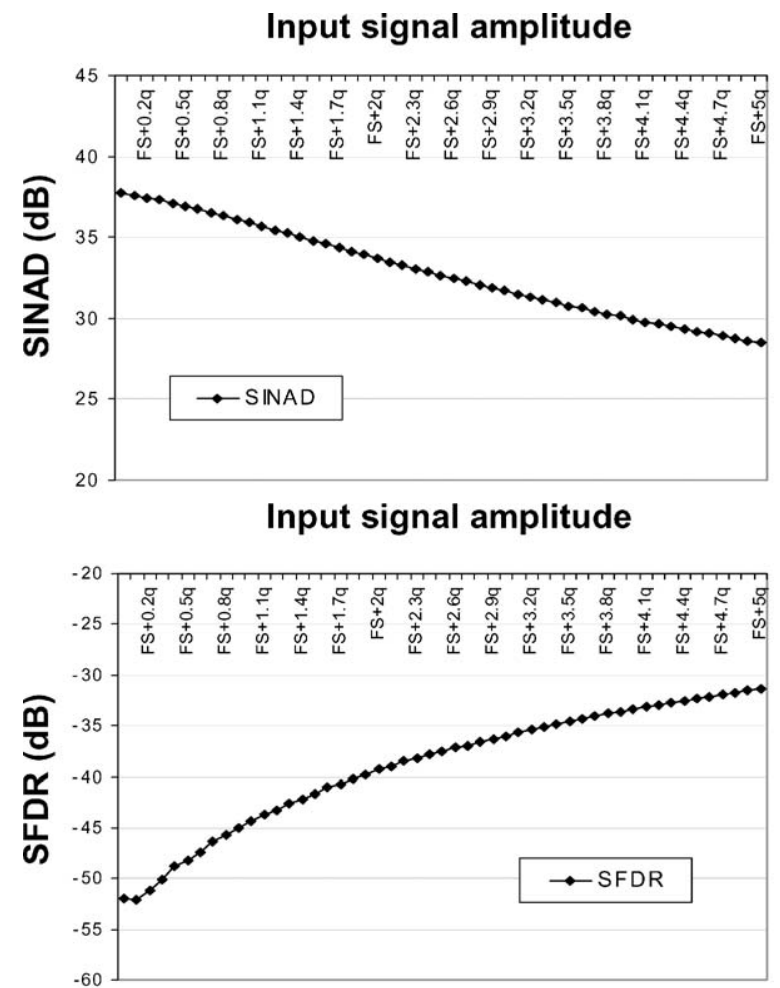

Input signal amplitude

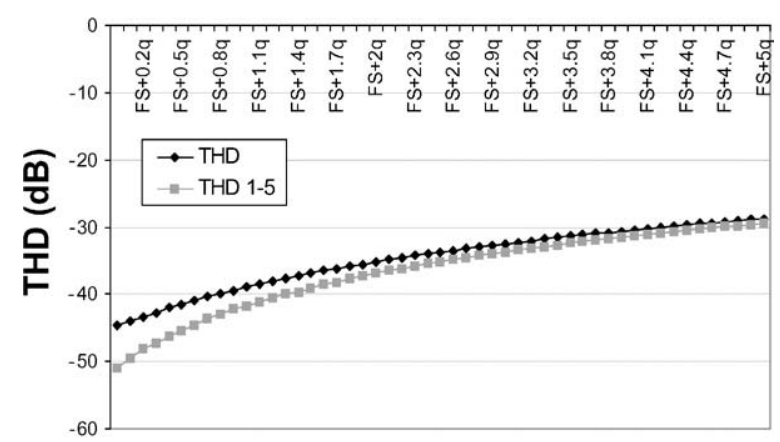

Fig. 5. Dynamic parameters vs. signal amplitude $\left(2 A_{\text {in }} \geq F S\right)$.

higher than $F S$, since relative accuracy achieved in both cases may differ by more than one order of magnitude.

\subsection{Influence of an Offset Error}

First, we consider the offset error influence. Fig. 6gives the relative deviation between the measured and ideal values of the SINAD, SFDR and $T H D_{1-5}$ parameters in presence of an offset error. Three different input signal peak-to-peak amplitudes have been considered, corresponding to $F S-2 L S B, F S$ and $F S+2 L S B$. The first 

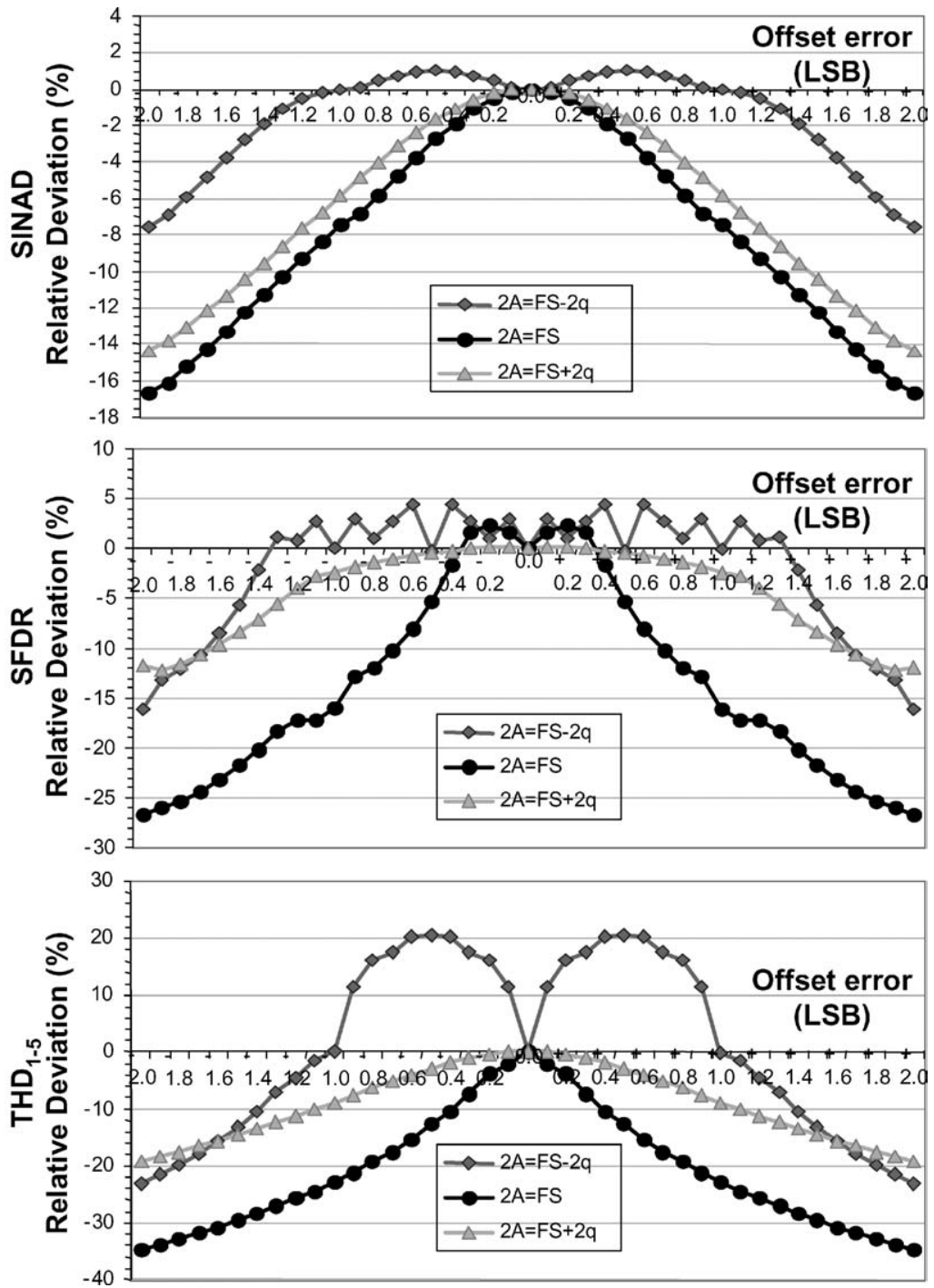

Fig. 6. Dynamic parameter relative deviations vs. offset error.

comment on these results is that an offset error significantly affects dynamic parameters in many cases, therefore opening the way of a possible detection of offset errors through the measurement of dynamic parameters. Indeed, the expected value of the dynamic parameters for an ideal converter is known from the previous study. Any difference between the measured and expected value indicates the converter is affected by an error.

Analyzing more in details the evolution of the dynamic parameters versus the offset error, it should be noted that two different behaviors are observed depending whether the input signal peak-to-peak amplitude is lower or higher than $F S$. We observe monotonous variation of the dynamic parameters with the offset error when the amplitude is higher than $F S$, which is not the case for an amplitude lower than FS. Such a monotonous variation is of great interest for the determination of the offset error without ambiguity. For instance, the relative deviation of the SINAD and THD parameters increases almost linearly with the offset value for an amplitude higher than $F S$, which permits a very straightforward evaluation of the offset error. Hence, applying an input signal larger than the full scale range of the converter seems more adequate for the detection of offset errors in a first view. 
Besides, the analysis of the variations of the dynamic parameters with respect to their sensitivity to the signal amplitude reveals that offset error detection is possible only in case of a signal amplitude higher than FS. Indeed the sensitivity of the dynamic parameters with respect to the signal amplitude is reduced in this case, allowing measurements accurate enough to discriminate an offset error from an amplitude deviation. On the contrary, variations induced by an offset error when applying a signal amplitude lower than $F S$ remain in the range of variations observed in case of a small deviation of the signal amplitude, preventing discrimination. For illustration, let us assume that the signal amplitude can be guaranteed within \pm 0.1 LSB around the nominal value. In case of an amplitude 2 LSB lower than $F S$, expected values for the SINAD, SFDR and $T H D_{1-5}$ can be determined with an accuracy of $1.6 \%, 4.6 \%$ and $50 \%$ respectively, as established in the previous section. Reporting these values on the corresponding characteristics of Fig. 6 shows that the SINAD and SFDR measurements permit to detect offset errors only if higher than 1.4 LSB and 1.5 LSB, while the poor accuracy obtainable on the $T H D_{1-5}$ measurement prevents from the detection of offset errors as high as 2 LSB. In case an amplitude 2 LSB higher than $F S$, expected values for the SINAD, SFDR and $T H D_{1-5}$ are known with an accuracy of $1.3 \%, 2 \%$ and $2.2 \%$ respectively, which leads to the detection of offset errors as low as $0.5 \mathrm{LSB}$, $1 \mathrm{LSB}$ and $0.2 \mathrm{LSB}$ respectively.

\subsection{Influence of a Gain Error}

Considering now a gain error, the evolution of the SINAD, SFDR and $T H D_{1-5}$ parameters is reported inFig. 7 for the three values of the input amplitude. While the evolution of dynamic parameters was perfectly symmetrical in case of positive and negative offset error, the situation is different with a gain error. Indeed, a negative gain error means that the effective ADC full scale is smaller than supposed, which induces a greater clamping of the converted signal. On the contrary, a positive gain error implies that the full scale is larger than expected; consequently the input signal will cover less codes then expected. In this latter case, we find a sensitivity similar to the one upon the signal amplitude.

Despite this difference, similar conclusions as for the detection of an offset error can be derived. In particular, we find out that the use of an input signal amplitude higher than the full scale of the ADC permits the evaluation of the gain error in a large range. As an example, gain errors of \pm 0.2 LSB can be detected from the SINAD measurement, $\pm 0.2 \mathrm{LSB}$ from the SFDR measurement and \pm 0.2 LSB from the $T H D_{1-5}$ measurement. In case of a signal amplitude lower than $F S$, detection of gain error is restricted to -2.7 LSB from the SINAD measurement and to -2.5 LSB from the $S F D R$ measurement, while the high sensitivity of the $T H D_{1-5}$ to the signal amplitude again prevents the detection of gain errors as high as \pm 4 LSB.

\subsection{Influence of Non-Linearity Errors}

Finally, the evolution of the dynamic parameters in presence of non-linearity errors has been investigated. While offset and gain errors can be clearly and fully defined by a value, non-linearity errors require to be cautiously considered. Indeed, only the maximal value of the non-linearity among the ADC codes is usually taken into account, but several types of non-linearity errors may be expressed by the same maximal value. Restricting the description of a non-linearity to its maximum value leads to a lack of information, as different types of non-linearity errors with the same peak values will not have the same impact on the dynamic parameters. Therefore, various cases of non-linearity have been considered.

To begin with, a unique integral non-linearity (INL) has been introduced in the transfer curve of the ADC model, on each code of a 6 bit ADC successively. For illustration, Fig. 8 givesthe results observed for an increasing positive INL value located exclusively on the tenth code. The study is restricted to INL values comprised between 0 and 1 LSB as a greatest value would imply a missing code.

The analysis of these results reveals a relatively different behavior of the dynamic parameters than when considering offset or gain errors. Indeed, we observe in this case a monotonic variation of the dynamic parameters both for a signal amplitude lower and higher than $F S$, but with a higher sensitivity for a signal amplitude lower than FS. Introducing the measurement uncertainty in the analysis, it arises that the use of a signal amplitude lower than $F S$ leads to better detection results for the dynamic parameters. Hence, in contrast with offset or gain detection results, it appears more performing to use an input signal with amplitude lower than FS. Further simulations considering more complex shapes of INL enable to generalize this observation to any INL influence. Far from making up 

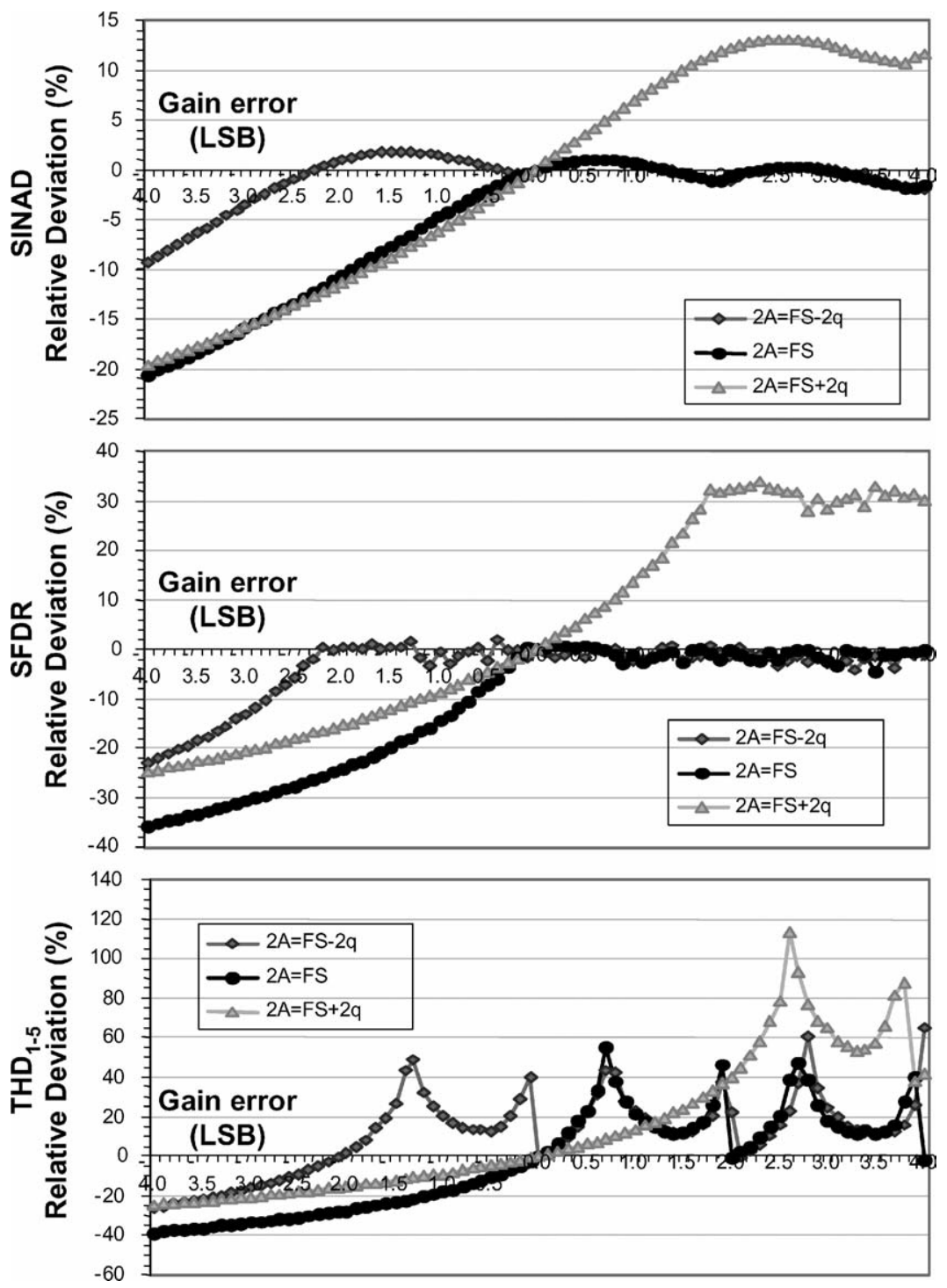

Fig. 7. Dynamic parameter relative deviations vs. gain error.

an impediment to the evaluation of static parameters from the deviation of the dynamic parameters, this increased sensitivity upon static faults under different conditions may outline a test strategy. Indeed, it would be of great interest to take advantage of both test conditions in terms of input signal amplitude to determine as precisely as possible each distinct static influence. Though the $T H D$ is more sensitive to non-linearity errors, it would be preferable to consider only the SINAD in the case of an amplitude lower than FS. Indeed, under this test condition, the THD measurement uncertainty is considerable and prevents to determine precisely static influence. Afterwards, we will only consider the SINAD parameter variation.

Comparing the respective impact of an integral nonlinearity on each code successively, a weighting effect appears depending on which code is affected. In particular, the presence of a non-linearity on an extreme code (one of the first or one of the last codes) induces a much more noticeable relative deviation on the extracted dynamic parameters than an INL of the same value located on a middle code.

Fig. 9 showsthe influence of an isolated INL on the SINAD measurement of a 6 bit ADC with respect to the 

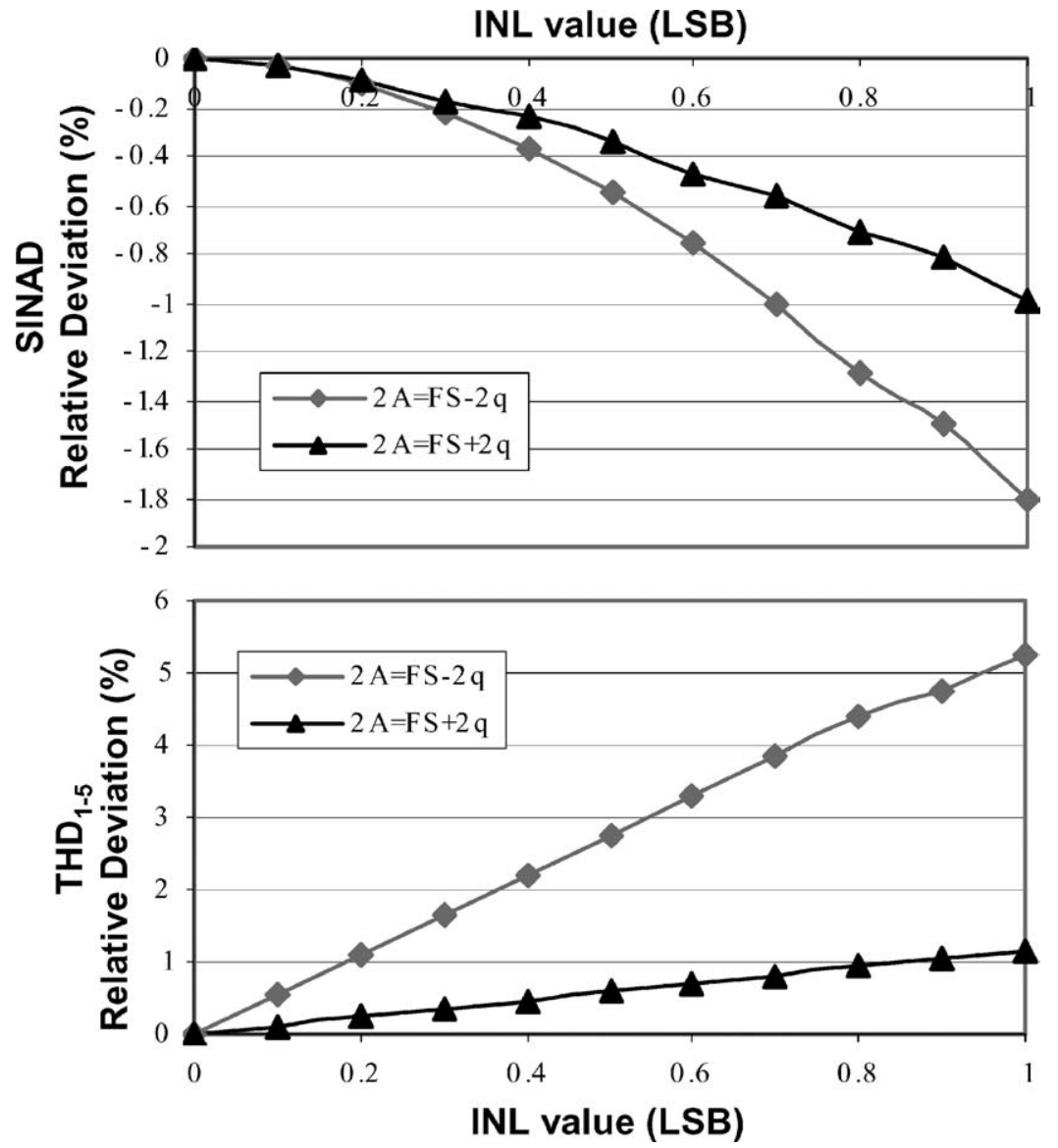

Fig. 8. Dynamic parameter relative deviations due to a unique INL on the tenth code.

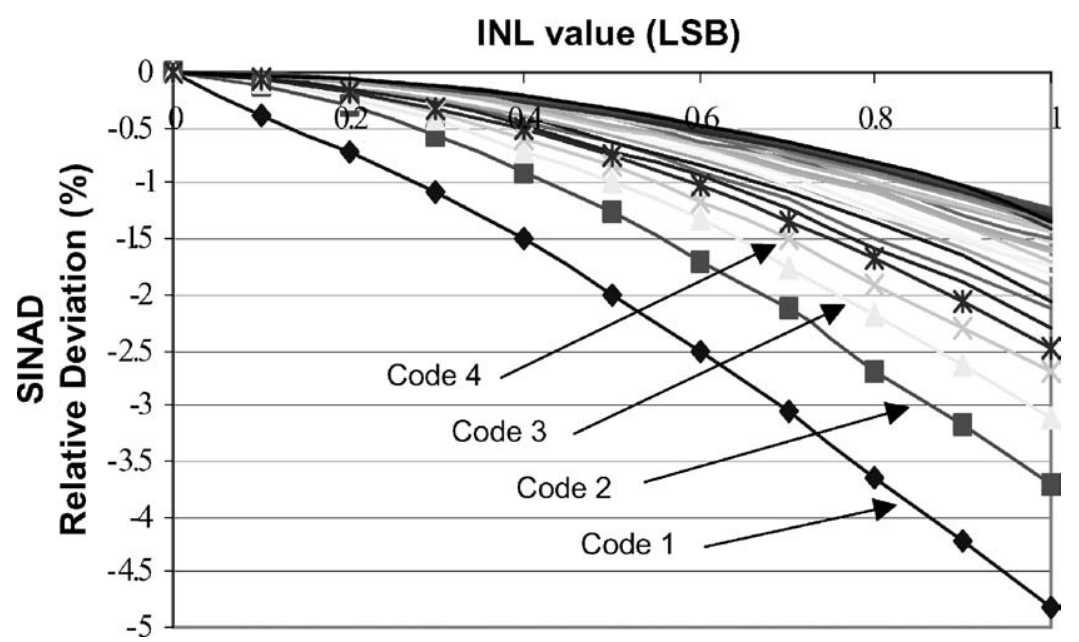

Fig. 9. SINAD relative deviation due to an INL affecting a unique code, from code 1 to code 32 . 

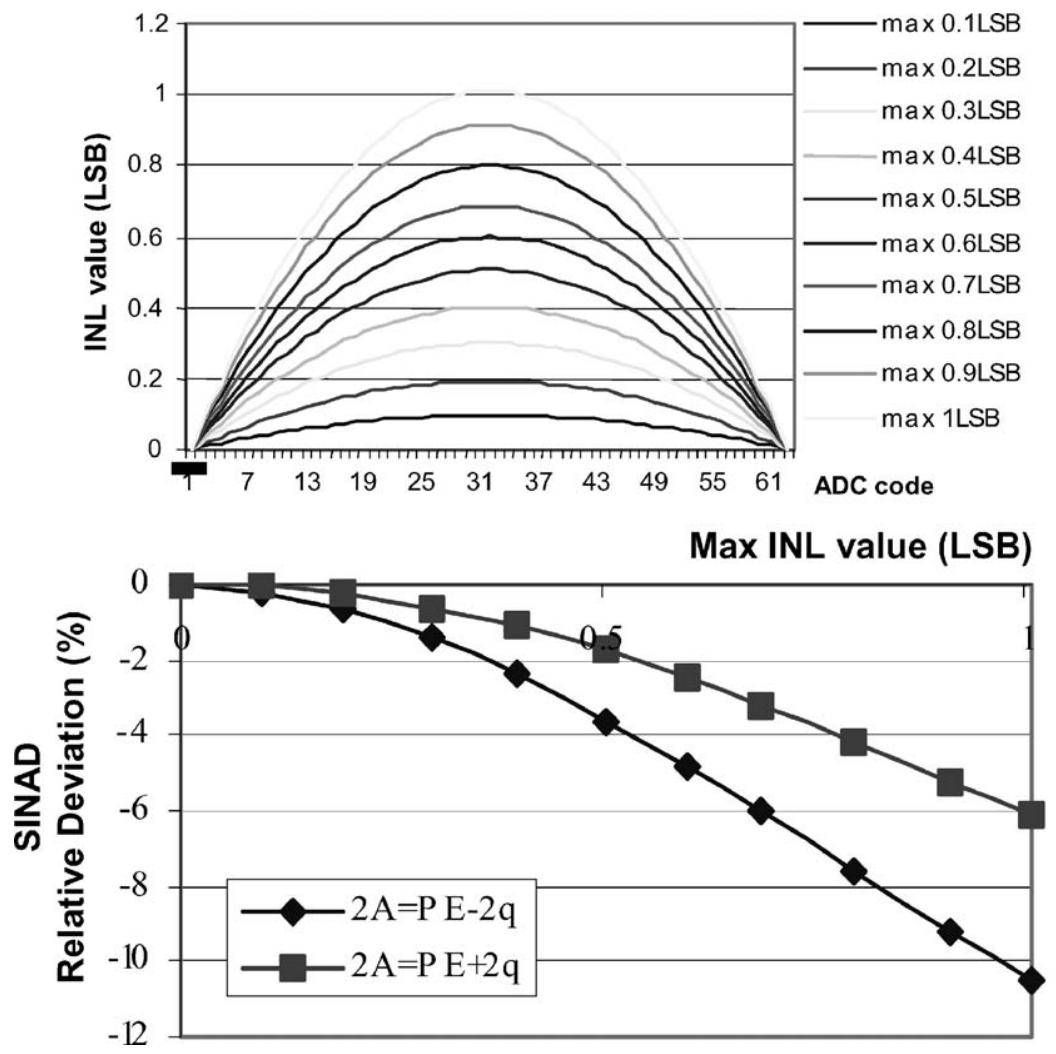

Fig. 10. Parabolic INL \& its influence on the SINAD.

introduced INL value with an input signal amplitude 2 LSB lower than FS. Each curve corresponds to a different INL position, varying from code 1 to code 32 . Note that a unique INL, with a value inferior to $1 \mathrm{LSB}$, leads to a minor global non-linearity. Therefore, the relative deviations observed in this study case remain in a hardly detectable range. We will see hereafter that a more realistic INL model leads to more significant deviation values.

The greatest deviation of the SINAD parameter is observed when an INL is introduced on code 1, and the impact of a given INL decreases according to its location in the ADC transfer curve up to code 32 . The sensitivity of the dynamic parameters to the INL influence, depending on the position of the affected code, is linked to the input signal sinusoidal shape. Indeed, due to the steepest slope nearby the signal mean value, the middle codes less affect the ADC response than the other codes. This explains why their impact on the ADC output spectrum and the related dynamic parameters is lessen in comparison with the influence of the other codes.
The second part of the non-linearity influence study takes into consideration some realistic INL types. Any shape of INL along the ADC codes can be modeled with a polynomial expression. In a first step, we analyze the influence of a parabolic INL, with a maximum value ranging between 0 and 1 LSB. The INL error and the induced relative deviation of the dynamic parameters areshown Fig. 10.

As already observed in the case of a unique INL isolated on one code, the sensitivity of the measured parameters is higher when the input signal amplitude is inferior to the ADC full scale. This result can be generalized to all the INL shapes.

Comparing the relative deviation of the SINAD induced by a single non-linearity on a code with the one corresponding to an ADC affected by a parabolic INL, it is obvious that we should not take into account only the maximal value to describe an INL in the perspective of non-linearity detection. Indeed, if the maximal value was a data complete enough to describe an INL, we should obtain the same SINAD deviation, at a given maximal INL value, in case of a unique 


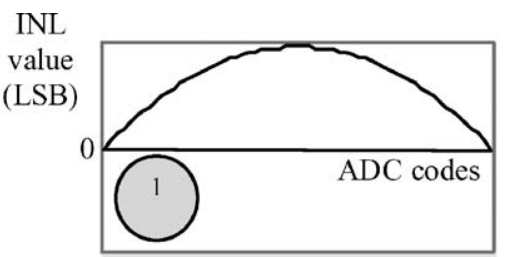

INL
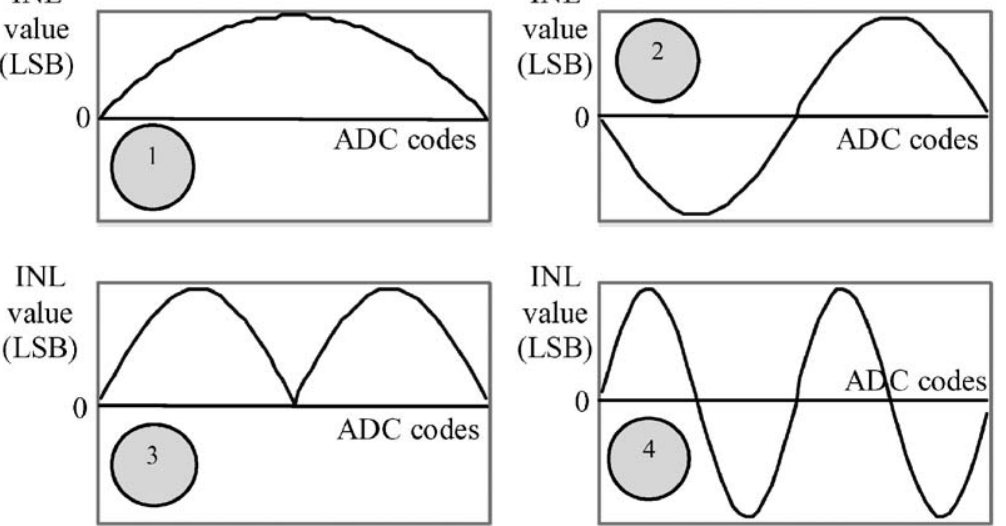

Max INL value (LSB)

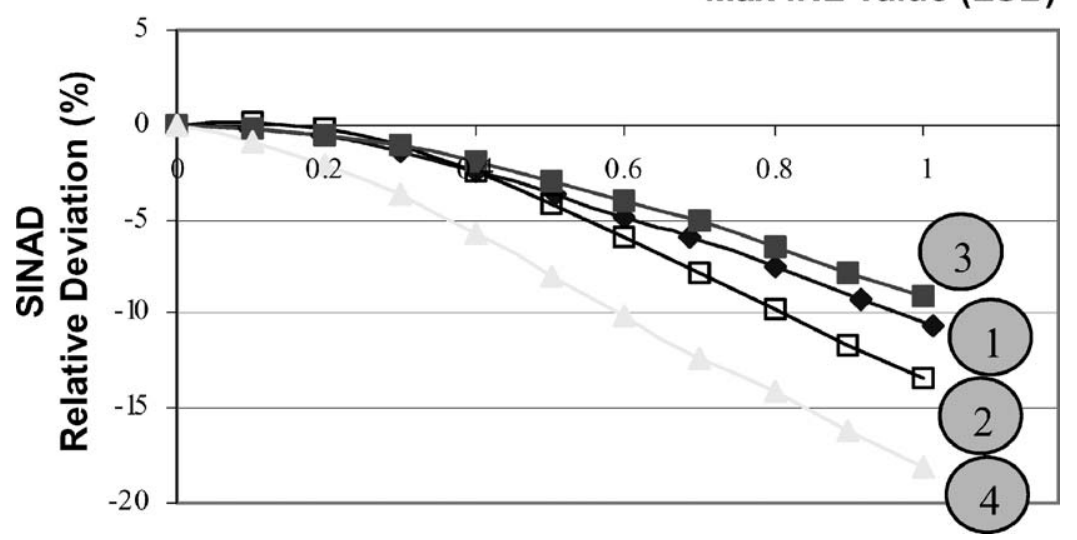

Fig. 11. Several INL shapes and their influence on the SINAD.

INL as in case of a parabolic one. On the contrary, we can clearly see that the different INL values along the ADC codes have a cumulative effect on the dynamic parameters. As a result, the SINAD parameter exhibits significant deviations in presence of a parabolic INL, even for a maximal INL value lower than 1 LSB.

Additional experiments have been conducted considering other shapes of INL. Fig. 11 representssome INL shapes and the relative deviations they induce on the SINAD parameter. Results are coherent with the observations made in the case of a parabolic INL: a cumulative effect of the INL contributions at the code level is observed, resulting in significant deviations of the SINAD parameter. It should be noted that the observed deviation for a given maximal INL value depends on the INL shape. However, it has not been possible so far to establish a simple relation between the INL contribution at the code level and the global INL shape.

\section{Conclusion}

In this paper, we have investigated the correlation between ADC static and dynamic parameters. The objective is to determine whether static errors could be characterized as well as dynamic performances from a spectral analysis. This would permit to avoid the histogrambased approach classically used for static evaluation.

In the first part of the study, the influence of the experimental setup on the measurement of the dynamic parameters has been considered. It appears that the dynamic parameters are not sensitive to the number of samples nor to the number of input periods but to the signal amplitude. This is particularly true for the $T H D$ measurement in case of a classical input signal with an amplitude slightly lower than FS. A measurement uncertainty has been associated to each dynamic parameter taking into account that it is extremely difficult to precisely adjust the signal amplitude in a real environment. 
The second part of the study has been dedicated to the influence of static errors on the measurement of the dynamic parameters. It appears that under appropriate test conditions, the dynamic parameters exhibit significant variations against static errors, allowing the detection of errors as low as some few tenths of LSB. In particular, it has been established that, although conventional testing uses an input signal with amplitude slightly lower than $F S$, the use of an input signal with amplitude slightly higher than $F S$ enhances the detection of both offset and gain errors. At the opposite, the detection of non-linearity errors is more performing using an input signal with amplitude lower than FS.

\section{References}

1. P. Carbone and D. Petri, "Effect of Additive Dither on the Resolution of Ideal Quantizers," IEEE Transactions on Instrumentation and Measurement, vol. 43, no. 3, pp. 389-396, 1994.

2. N. Csizmadia and A.J.E.M. Janssen, "Estimating the Integral Non-Linearity of AD-Converters via the Frequency Domain," in Proc. International Test Conference, 1999, pp. 757-762.

3. R. de Vries and A.J.E.M. Janssen, "Decreasing the Sensitivity of ADC Test Parameters by means of Wobbling," Journal of Electronic Testing: Theory and Applications, vol. 15, pp. 23-29, 1999.

4. J. Doernberg, H.S. Lee, and D.A. Hodges, "Full-Speed Testing of A/D Converters," IEEE Journal of Solid State Circuits, vol. 19, no. 6, pp. 820-827, 1984.

5. European Project DYNAD, "Methods and draft standards for the DYNamic characterization and testing of Analog-toDigital converters," published on the web at http://www.fe. up.pt/ hsm/dynad/

6. T. Kuyel, "Linearity Testing Issues of Analog to Digital Converters," in Proc. International Test Conference, 1999, pp. 747-756.

7. M. Mahoney, "DSP-Based Testing of Analog and Mixed-Signal Circuits," IEEE Computer Society Press, ISBN 0-8186-0785-8, 1987.

8. H.S. Mendonça, J.M. Silva, and J.S. Matos, "A Comparison of ADC Dynamic Test Methods," in Proc. Design of Circuits and Integrated Systems Conference, 2000, pp. 102-107.

9. Y. Yenq, "Measuring Harmonic Distortion and Noise Floor of an A/D Converter Using Spectral Averaging," IEEE Transactions on Instrumentation and Measurement, vol. 37, pp. 525-528, 1988.

Florence Azaïs received M.Eng., and Ph.D. degrees in electrical engineering from the University of Montpellier, France in 1993 and 1996, respectively. She is currently working in the Microelectronics department of the Laboratory of Computer Science, Robotics and Microelectronics of Montpellier (LIRMM) as a researcher of the
National Council of Scientific Research (CNRS). Since 1993, she has been interested in the general domain of integrated circuit testing. She has authored or co-authored more than 80 international papers. Her main research interests include fault modeling and mixed-signal circuit testing, and in particular design-for-testability and built-in self-test techniques.

Serge Bernard received the M.S. degree in Electrical Engineering from the University of Paris XI, France in 1998 and the Ph.D. degree in Electrical Engineering from the University of Montpellier, France in 2001. He is a researcher of the National Council of Scientific Research (CNRS) in the Microelectronics Department of the Laboratory of Computer Science, Robotics and Microelectronics of Montpellier (LIRMM). His main research interests include Test, Design-For-Testability and Built-In-Self-Test for mixed-signal circuits and Design-For-Reliability for medical application ICs.

Yves Bertrand is a professor at the University of Montpellier (France). He works at the Microelectronics Department of the Laboratory of Computer Science, Robotics and Microelectronics of Montpellier (LIRMM). Previously, Yves BERTRAND worked in the field of solid-state physics and published several papers, especially on the photoemission of the semiconductors under synchrotron radiation. He joined the LIRMM in 1988. His research interests are principally Fault Modeling, Design-For-Test and Built-In-Self-Test for digital and mixed-signal Integrated Circuits and Design \& Test of Microsystems. He is author or co-author of more than 170 papers in the field of solid-state physics and microelectronics. He is presently responsible for the CRTC (Test Resource Center at European level) and coordinator of the European EuNicE Test IST project.

Mariane Comte took her Master of Engineering and Master of Sciences degrees in microelectronics engineering at INPG, (Institut National Polytechnique de Grenoble, National Engineering University Institution of Grenoble), France, in 2000. She carried out her Ph.D. studies at LIRMM (Laboratoire d'Informatique, de Robotique et de Microélectronique de Montpellier, Computer Sciences, Robotics and Microelectronics Laboratory of Montpellier), France, working on Analog-to-Digital Converter testing, and received Ph.D. degree in microelectronics from the University of Montpellier, France, in 2003. She is currently working on Domino Cell testing at the Computer Design and Test Laboratory of NAIST (Nara Institute of Science and Technology), Japan, as a post-doctoral fellow of the COE program.

Michel Renovell is head of the Microelectronics Department at LIRMM (Laboratory of Computer Science, Automation and Microelectronics of Montpellier). His research interests include: Fault modeling, Analog testing and FPGA testing. He is Vice-Chair of the IEEE TTTC (Test Technology Technical Committee), Chair of the Communication Group and Chair of the FPGA testing Committee. He is a member of the editorial board of JETTA and the editorial board of IEEE Design \& Test. Michel has been General Chair of several conferences: International Mixed Signal Testing Workshop IMSTW2000, Field Programmable Logic Conference FPL2002; He will serve as general Chair of the European Test Symposium ETS2004. 